\title{
Hydrophilic monomers suppress the adsorption of plasma protein onto a poly(vinylidene fluoride) membrane
}

\author{
AKIRA TAKAHASHI and HISASHI HISATOMI \\ Laboratory of Cellular and Molecular Biochemistry, Department of Materials and Life Science, \\ Seikei University, Tokyo 180-8633, Japan
}

Received December 19, 2008; Accepted June 24, 2009

DOI: $10.3892 / \mathrm{mmr} \_00000167$

\begin{abstract}
Dialysis is the single most important therapy for chronic kidney disease. However, protein adsorption onto hemodialysis membranes promotes clot formation. The aim of the present study was to develop a surface-modified membrane which suppresses protein adsorption. Using plasma polymerization, hydrophilic N-vinyl pyrrolidone (VP) and acryloyl morpholine (ACMO) were polymerized on hydrophobic poly(vinylidene fluoride) (PVDF) surfaces. PVDF is employed as a biomaterial owing to its extraordinary durability. These membranes were then characterized using static contact angle measurement, ATR-FTIR spectra and polyacrylamide gel electrophoresis. The surface-modified PVDF membranes showed a transition of the surface from relatively hydrophobic to hydrophilic. Less proteins were adsorbed on the VP and ACMO grafted PVDF surfaces than on the unmodified membrane and the commercially available hemodialysis membrane. In conclusion, VP and ACMO grafted PVDF membranes have a high potential for the inhibition of protein adsorption in high-performance hemopurification devices.
\end{abstract}

\section{Introduction}

The improvement of membranes used for the treatment of chronic kidney disease is an important issue (1). The ideal surface of an artificial blood purification membrane requires hemocompatibility and durability with high performance; such membranes should not adsorb any proteins or cells, but

Correspondence to: $\mathrm{Dr}$ Hisashi Hisatomi, Laboratory of Cellular and Molecular Biochemistry, Department of Materials and Life Science, Seikei University, 3-3-1 Kichijoji Kita-machi, Musashino-shi, Tokyo 180-8633, Japan

E-mail: hisatomi@st.seikei.ac.jp

Abbreviations: PVDF, poly(vinylidenefluoride); DMAA, N,Ndimethylacrylamide; VP, N-vinyl pyrrolidone; ACMO, acryloyl morpholine; ATR-FTIR, attenuated total reflection fourier transform infrared

Key words: dialysis, hemopurification, hydrophilic monomer, surface modification should still have high permeability for the desired range of solute sizes. Recently, dialysis membranes have undergone continuous evolution. Their basic structure has been modified to improve the biocompatibility profile of the material and of membranes based on synthetic polymers $(2,3)$. Cellulose membranes, used commonly as hemopurification membranes, contain hydroxy groups, which induce leukocyte activation and enhance inflammation (4). Synthetic polymers used in hemopurification are predominantly hydrophilic and adsorb most proteins. This adsorption also induces inflammation and/or clot formation (5). In addition, protein adsorption to the hemopurification membrane not only leads to leukocyte activation, but also decreases permeability and reduces membrane performance. Therefore, synthetic polymers require continuous improvement in terms of hemocompatibility, with enhanced suppression of protein adsorption and clot formation. To improve the anti-fouling property of hemopurification membranes, researchers have developed various strategies that may control hydrophilia in hydrophobic synthetic polymer materials at the structural and surface-active levels (6-8).

Commercially available hemodialysis membranes, such as polysulfone membanes, are based on hydrophilic materials, with some suppression activity on protein adsorption. A potential problem involving these commercially available membranes is their durability. To avoid this problem and yet decrease protein adsorption, we modified the surface of a membrane made of hydrophilic poly(vinylidene fluoride) (PVDF), which has higher heat and chemical stability than polysulfone, as the base of a hemopurification membrane. PVDF is commonly used as a biomaterial due to its non-toxicity and extraordinary durability (7). Hydrophobic PVDF membranes are not capable of suppressing protein adsorption to their surface; however, we hypothesized that a surface-modified PVDF membrane might effectively prevent protein adsorption. Thus, we introduced three types of hydrophilic monomers to the PVDF surface: N,N-dimethylacrylamide (DMAA), N-vinyl pyrrolidone (VP) and acryloyl morpholine (ACMO). DMAA has been commonly used as a biomaterial for contact lenses $(9,10), \mathrm{VP}$ is a typical biomaterial $(11,12)$ and ACMO has a VP-like structure $(13,14)$. These monomer chains were introduced by plasma polymerization, a technique that has been widely used for the surface modification of polymers as it involves localized surface treatment and does not change the bulk properties of the polymer (7). 
In this study, three surface-modified high-polymer membranes were evaluated for their protein adsorption characteristics and use as a hemopurification membrane device. Our results reveal the possibility of a new hemopurification membrane.

\section{Materials and methods}

Introduction of hydrophilic monomers to the membrane surface and measurement of water contact angles. All solvents and reagents, unless otherwise specified, were purchased from Wako Junyaku (Osaka, Japan) at the highest purity grade. PVDF hollow fiber membranes with an internal diameter of $0.75 \mathrm{~mm}$ and an external diameter of $1.3 \mathrm{~mm}$ were obtained from Asahi Chemical Corp. (Osaka, Japan). PVDF hollow fiber membranes were exposed to Ar plasma (10 min, $20 \mathrm{~W}$ ), and a hydrophilic monomer gas (DMAA, VP or ACMO) was introduced to the membrane surface for $60 \mathrm{~min}$. Membranes were washed with milli-Q water, then dried in a vacuum for $6 \mathrm{~h}$. Subsequently, the amount of the introduced monomer $\left(\mathrm{mg} / \mathrm{mm}^{2}\right)$ was calculated from the difference between the dry weight of each hollow fiber membrane before and after the introduction of each monomer. The surface area per $10 \mathrm{~mm}$ of the PVDF hollow fiber membrane, $64 \mathrm{~mm}^{2}$, was used as a reference. The grafted hydrophilic monomer was verified using attenuated total reflection fourier transform infrared (ATR-FTIR) spectroscopy (MIRacle ${ }^{\mathrm{TM}}$ Single Reflection HATR, Pike Technology, WI, USA). For water contact angle analysis, we introduced three types of hydrophilic monomers to the PVDF flat-sheet membranes (Millipore, CA, USA), as well as those mentioned above. The surface of the modified PVDF flat-sheet membrane was characterized by means of the static contact angle measurements.

Evaluation of protein adsorption using proteomic analysis. A polysulfone-membrane dialyzer (also known as APS ${ }^{\mathrm{TM}}$ ) was used as the control hollow fiber membrane $(15,16)$. Polysulfonemembrane dialyzers with an internal diameter of $0.2 \mathrm{~mm}$ were obtained from Asashi Kasei Medical Co. Ltd. (Tokyo, Japan). The three types of surface-modified PVDF membranes and the polysulfone-membrane dialyzer were arranged in the same surface area of $64 \mathrm{~mm}^{2}$. The PVDF hollow fiber membranes and the polysulfone-membrane dialyzer were cut at 10 and $45 \mathrm{~mm}$, respectively. Each hollow fiber membrane was immersed in $20 \mu \mathrm{l}$ of human whole blood derived from healthy volunteers at $37^{\circ} \mathrm{C}$ for $1 \mathrm{~h}$. Ethylenediaminetetraacetic acid (EDTA) was used to suppress blood clot formation. After the membranes were washed with phosphate-buffered saline (137 mM NaCl, $2.7 \mathrm{mM} \mathrm{KCl}, 1.5 \mathrm{mM} \mathrm{KH}_{2} \mathrm{PO}_{4}$ and $8.1 \mathrm{mM}$ $\mathrm{NaHPO}_{4}$ ), total protein was extracted using $20 \mu \mathrm{l}$ extraction buffer \{0.1 M 2-mercaptoethanol, 0.5\% 3-[(3-cholamidopropyl) dimethylammonio]-1-propanesulfonate (CHAPS), $10 \%$ glycerine, $1 \mathrm{mM}$ EGTA, $1 \mathrm{mM} \mathrm{MgCl}_{2}$ and $0.1 \mathrm{M}$ Tris- $\mathrm{HCl}$ $\mathrm{pH} 7.5\}$. Half of the extracted protein was separated using sodium dodecyl sulfate polyacrylamide gel electrophoresis (SDS-PAGE) with 5-20\% gradient polyacrylamide gel (Atto, Tokyo, Japan) and was stained with Coomassie brilliant blue (Bio-Rad, Hercules, CA, USA). Each signal was detected using a charge-coupled device camera in a VersaDoc 4000 System (Bio-Rad).

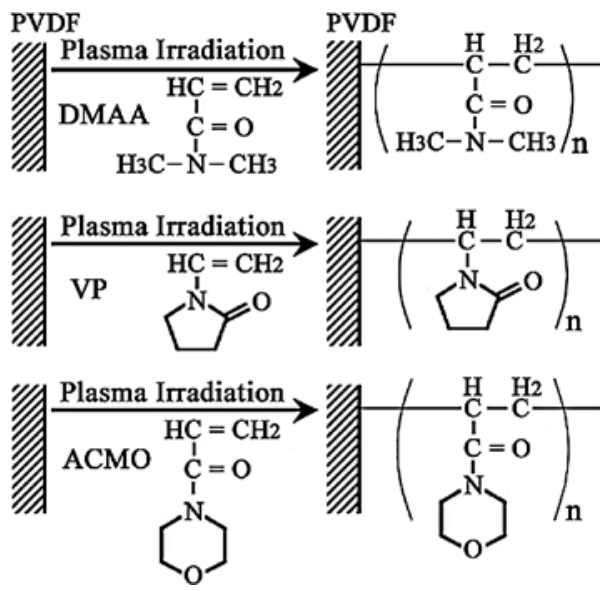

Figure 1. Reaction scheme for the plasma polymerization modified PVDF membrane surface.

Protein signals on the stained polyacrylamide gels were excised and digested with $0.4 \%$ (w/v) trypsin overnight. The digested peptides were detected using a matrix-assisted laser desorption/ionization time-of-flight mass spectrometry (MALDI-TOF MS, Axima ${ }^{\circledR}$-CFR plus; Shimadzu, Kyoto, Japan). Database searches were carried out using the Mascot Search engine in Matrix Science (http://www.matrixscience. com/) (17).

\section{Results}

Introduction of hydrophilic monomers to the membrane surface and measurement of water contact angles. Fig. 1 shows a schematic illustration of surface modifications by graft copolymerization of each monomer. The ATR-FTIR spectrum of the DMAA grafted PVDF membranes showed the characteristic $\mathrm{C}=\mathrm{O}$ stretching band of the amide group derived from the DMAA monomer, which was not observed in the unmodified PVDF membrane or the PVDF membrane after plasma treatment (Fig. 2). The ATR-FTIR spectra of the VP and ACMO grafted membranes had similar wavelengths as the respective monomer origins (Fig. 2).

Compared with the unmodified PVDF hollow fiber membrane, the dry weights of the DMAA $(n=5)$, VP $(n=5)$ and ACMO grafted $(n=4)$ membranes increased by $47.5 \pm 3.9$, $7.4 \pm 1.7$ and $14.6 \pm 2.4 \mu \mathrm{g} / \mathrm{mm}^{2}$, respectively. The static contact angles of the DMAA, VP and ACMO grafted membranes were $18 \pm 2.9,24 \pm 2.1$ and $29 \pm 7.3^{\circ}$, respectively. Compared with the unmodified PVDF membrane with a contact angle of $84 \pm 2.5^{\circ}$, all surface modifications led to an increase in wettability with water. These data are the means of three measurements.

Evaluation of protein adsorption using proteomic analysis. Fig. 3 shows the adsorption of plasma protein to the surfacemodified membranes. The amount of protein adsorption on the unmodified PVDF, Ar plasma-treated, DMAA grafted PVDF, VP grafted PVDF, ACMO grafted PVDF and polysulfone (APS) membranes was 40.9 $\pm 6.6,54.1 \pm 7.7,49.3 \pm 8.7,17.5 \pm 4.6$, $18.3 \pm 3.5$ and $68.7 \pm 5.0 \mu \mathrm{g}$, respectively. These data are the means of three independent experiments. The amount of protein 


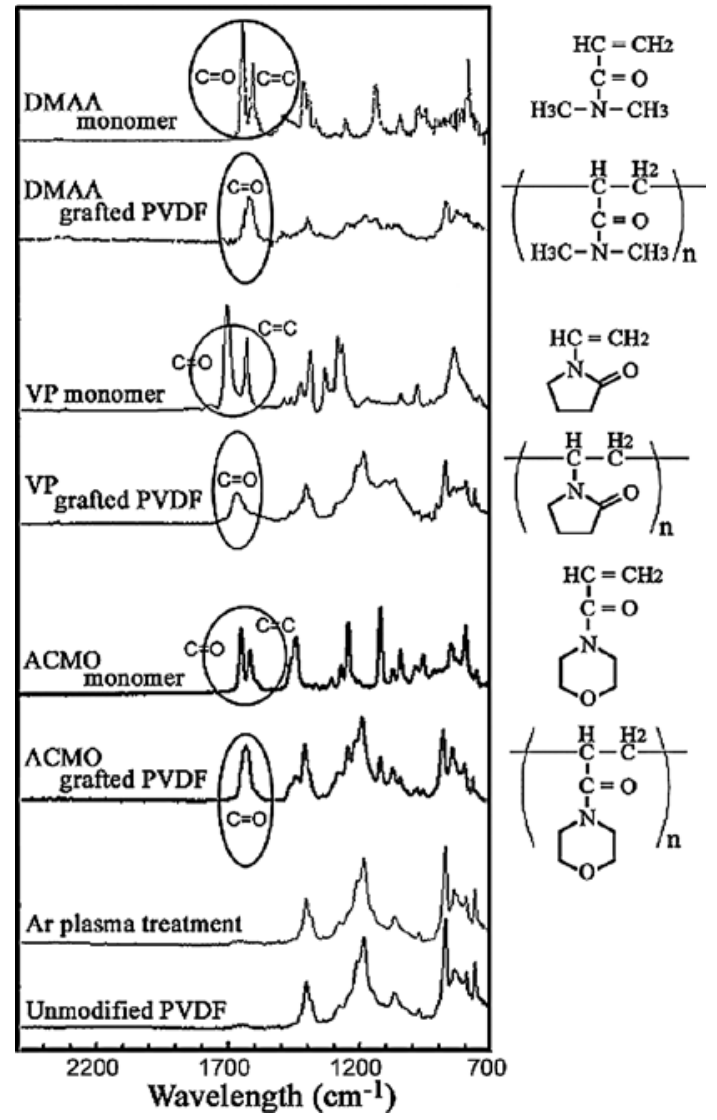

Figure 2. Overlay of ATR-FTIR spectra. DMAA, VP and ACMO monomers: infrared spectrum of each monomer with a $\mathrm{C}=\mathrm{O}$ stretching band in a vinyl group and a $\mathrm{C}=\mathrm{C}$ stretching band in an amide group. DMAA, VP and ACMO grafted PVDF: infrared spectrum of each grafted PVDF hollow fiber membrane. Ar plasma treatment: infrared spectrum of PVDF hollow fiber membrane with Ar plasma exposure. Unmodified PVDF: infrared spectrum of unmodified PVDF hollow fiber membrane.

adsorbed onto the VP and ACMO modified surface was markedly decreased compared with that adsorbed onto the unmodified surface $(\mathrm{p}<0.05)$, whereas the amount of protein adsorbed onto the DMAA modified surface was unchanged. The VP and ACMO grafted PVDF surfaces exhibited less protein adsorption than the commercially available hemodialysis membranes, such as the polysulfone-membrane dialyzer $(\mathrm{p}<0.05)$.

Three proteins adsorbed to the surface of the membranes used in this study were identified. These were transferrin at $79 \mathrm{kDa}$, serum albumin at $68 \mathrm{kDa}$ and carbonate dehydratase at $28 \mathrm{kDa}$. VP and ACMO grafted PVDF hollow fiber membranes suppressed the adsorption of all the identified proteins. Identified proteins that could have resulted from artifacts were excluded, since triplicate experiments based on independent membranes yielded consistent results.

\section{Discussion}

In the present study, we confirmed the introduction of monomers to PVDF surfaces by ATR-FTIR, as well as the transition of the PVDF surface from relatively hydrophobic to hydrophilic by a decrease in the static contact angle on the surface-modified membrane. Hydrophilicity was expected to be significantly reduced for the modified surfaces. Analysis

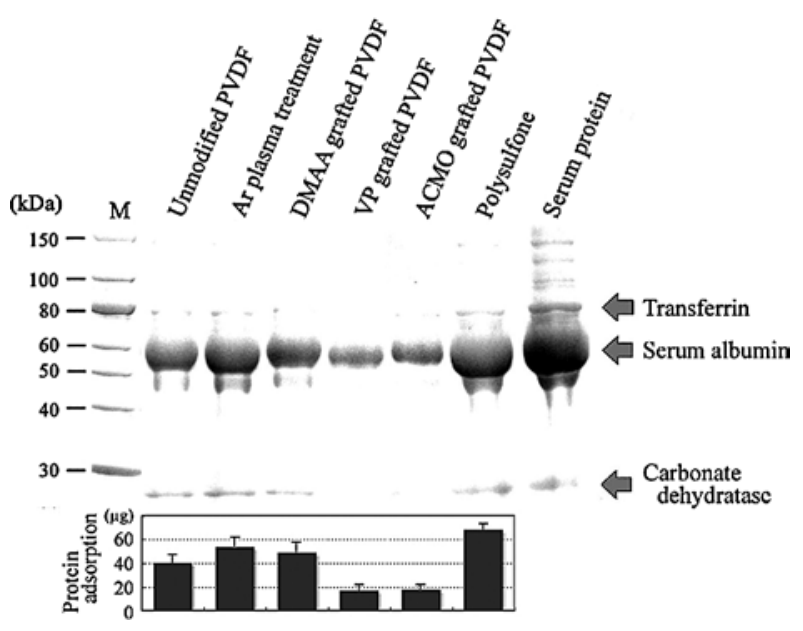

Figure 3. Adsorption of protein in whole blood onto surface-modified membranes. M, protein size maker; unmodified PVDF, unmodified PVDF hollow fiber membrane; Ar plasma treatment, PVDF hollow fiber membrane with Ar plasma exposure; DMAA grafted PVDF, DMAA grafted PVDF hollow fiber membrane; VP grafted PVDF, VP grafted PVDF hollow fiber membrane; ACMO grafted PVDF, ACMO grafted PVDF hollow fiber membrane; polysulfone, polysulfone-membrane dialyzer; serum protein, serum in whole blood with EDTA.

of the contact angles confirmed this prediction, and also confirmed that the static contact angles were different due to differences in the grafted monomers. Of the three types of hydrophilic monomers used, the static contact angle was markedly decreased for DMAA. However, the DMAA grafted PVDF did not exhibit the suppression of protein adsorption, whereas the DMAA grafted surface showed greater hydrophilicity than the VP and ACMO grafted surfaces. Adsorption of proteins occurs on a variety of solid-liquid interfaces. In many instances, protein adsorption results in undesirable effects, such as surface-induced clot formation, due to the adsorption of plasma proteins or the fouling of membranes used in food and beverage processing (18). Although various factors affect protein adsorption, the surface chemistry of the substrate has been extensively studied using a variety of surface modification techniques, with the goal of reducing protein adsorption. For instance, previous studies have demonstrated that the increase in the swollen area caused by DMAA on copolymers enhances albumin adsorption $(10,19)$. Our results are in close agreement with those of De Queiroz et al (10), who revealed that DMAA increases albumin adsorption. Moreover, in our study the DMAA grafted PVDF membrane not only adsorbed serum albumin, but also showed the adsorption of other proteins, such as transferrin and carbonate dehydratase. In contrast, both the VP and ACMO grafted PVDF effectively prevented protein adsorption. It is of interest that the amount of protein adsorption differed among the three types of monomer grafted membranes, while these membranes had similar hydrophilic ability. We also confirmed that a decrease in the amount of monomer grafted onto each PVDF surface (for example, VP or ACMO grafted for $2 \mathrm{~min}$ ) did not influence the amount of protein adsorption (data not shown). For surface-modified PVDF membranes, these results indicate that the characteristics of the monomer grafted to the membrane surface play a more important role in protein adsorption than the amount of monomer grafted or hydrophilic ability. 
VP and ACMO grafted PVDF hollow fiber membranes demonstrated stronger suppression of protein adsorption than commercially available hemodialysis membranes, such as polysulfone-membrane dialyzer. The amount of protein adsorption onto the VP and ACMO grafted PVDF membranes was suppressed by one-fourth compared with that adsorbed onto the commercially available hemodialysis membranes (17.5 \pm 4.6 and $18.3 \pm 3.5$ vs. $68.7 \pm 5.0)$.

All the detectable proteins adsorbed onto the VP and ACMO grafted PVDF membranes had similar molecular weights as plasma protein. Thus, it is unlikely that VP and ACMO membrane adsorption excluded plasma protein. Based on these results, it is unknown whether the two modified membranes would be as effective as commercially available hemodialysis membranes when put into actual use. However, we suggest that the suppression of protein adsorption using VP or ACMO grafted PVDF membranes may offer new treatment approaches for the control of hemopurification.

Our observation of a relationship between the type of monomer and protein adsorption may serve as an important model for the improvement of hemodialysis membranes, and may provide novel strategies to enhance hemopurification.

\section{References}

1. Hoenich NA: Membranes for dialysis: can we do without them? Int J Artif Organs 30: 964-970, 2007.

2. Qiu Y, Zhang N, An YH and Wen X: Biomaterial strategies to reduce implant-associated infections. Int $\mathrm{J}$ Artif Organs 30: 828-841, 2007.

3. Vienken J: Polymers in nephrology. Characteristics and needs. Int J Artif Organs 25: 470-479, 2002.

4. Hansch GM, Karnaoukhova S, Chang SH, et al: Activation of human neutrophils after contact with cellulose-based haemodialysis membranes: intracellular calcium signalling in single cells. Nephrol Dial Transplant 11: 2453-2460, 1996.

5. Hong J, Azens A, Ekdahl KN, Granqvist CG and Nilsson B: Material-specific thrombin generation following contact between metal surfaces and whole blood. Biomaterials 26: 1397-1403, 2005.
6. Ye SH, Watanabe J, Iwasaki Y and Ishihara K: Antifouling blood purification membrane composed of cellulose acetate and phospholipid polymer. Biomaterials 24: 4143-4152, 2003.

7. Singhal JP and Ray AR: Synthesis of blood compatible polyamide block copolymers. Biomaterials 23: 1139-1145, 2002.

8. Ademovic Z, Klee D, Kingshott P, Kaufmann R and Hocker H: Minimization of protein adsorption on poly(vinylidene fluoride). Biomol Eng 19: 177-182, 2002.

9. Soltys PJ and Etzel MR: In vitro characterization of a membranebased low-density lipoprotein affinity adsorption device. Blood Purif 16: 123-134, 1998.

10. De Queiroz AA, Gallardo A, Román JS and Higa OZ: Hemocompatible properties of polymeric derivative of paracetamol. J Biomater Sci Polym Ed 7: 523-530, 1995.

11. Garrett Q, Laycock B and Garrett RW: Hydrogel lens monomer constituents modulate protein sorption. Invest Ophthalmol Vis Sci 41: 1687-1695, 2000.

12. Rosenberg R, Gertler R, Friederichs J, et al: Comparison of two density gradient centrifugation systems for the enrichment of disseminated tumor cells in blood. Cytometry 49: 150-158, 2002.

13. Källtorp M, Carlén A, Thomsen P, Olsson J and Tengvall P: Analysis of rat plasma proteins desorbed from gold and methyland hydroxyl-terminated alkane thiols on gold surfaces. J Mater Sci Mater Med 11: 191-199, 2000.

14. Maeda Y, Nakamura T and Ikeda I: Change in solvation of poly (N,N-diethylacrylamide) during phase transition in aqueous solutions as observed by IR spectroscopy. Macromolecules 35: 10172-10177, 2002.

15. Bowry SK: Dialysis membranes today. Int J Artif Organs 25: 447-460, 2002.

16. Ronco $\mathrm{C}$ and Bowry S: Nanoscale modulation of the pore dimensions, size distribution and structure of a new polysulfone-based high-flux dialysis membrane. Int J Artif Organs 24: 726-735, 2001.

17. Belgacem O, Buchacher A, Pock K, et al: Molecular mass determination of plasma-derived glycoproteins by ultraviolet matrix-assisted laser desorption/ionization time-of-flight mass spectrometry with internal calibration. J Mass Spectrom 37: 1118-1130, 2002.

18. Rovira-Bru M, Giralt F and Cohen Y: Protein adsorption onto zirconia modified with terminally grafted polyvinylpyrrolidone. J Colloid Interface Sci 235: 70-79, 2001.

19. Sotiropoulou M, Bokias G and Staikos G: Water-soluble complexes through coulombic interactions between bovine serum albumin and anionic polyelectrolytes grafted with hydrophilic nonionic side chains. Biomacromolecules 6: 1835-1838, 2005. 